\title{
Effect of the external helical fields on the plasma boundary shape in JET.
}

\author{
D Yadykin ${ }^{1}, \mathrm{M}_{\text {Gryaznevich }}^{2,4}$, L Frasinetti $^{3}$, S Gerasimov ${ }^{2}$ and JET-EFDA contributors* \\ JET-EFDA, Culham Science Centre, Abingdon, OX14 3DB, UK \\ ${ }^{1}$ Euratom/VR Fusion Association, Chalmers University of Technology, Gothenburg, S-41296, \\ Sweden \\ ${ }^{2}$ Euratom/CCFE Fusion Association, Culham Science Centre, Abingdon, OX14 3DB, UK \\ ${ }^{3}$ Euratom/VR Fusion Association, Royal Institute of Technology KTH, SE-10044 Stockholm, Sweden \\ ${ }^{4}$ Technical University of Denmark, DTU Ris $\emptyset$, Roskilde, Denmark \\ * See the Appendix of F. Romanelli et al., Proceedings of the 24th IAEA Fusion Energy Conference \\ 2012, San Diego, US
}

\begin{abstract}
Externally applied helical magnetic fields are now often used on tokamaks for various purposes. This paper presents results of studies of the effect of the external fields, produced by the error field correction coils (EFCCs) on JET, on the plasma boundary shape. Significant 3D distortions, predicted in the previous studies, have been confirmed using upgraded magnetic diagnostics and high resolution Thomson Scattering (HRTS) diagnostics. A simple method of estimating the edge distortion using magnetic diagnostics calibrated on the kinetic measurements is proposed and demonstrated.
\end{abstract}

PACS numbers: 52.55.-s, 52.75.-d, 52.30.-q

\section{Introduction.}

Application of external magnetic perturbations is a useful tool to investigate plasma stability boundaries [1-4] and the resistive wall properties [5,6]. External helical magnetic fields are also used for error field correction [7] and for the direct suppression (feedback or mitigation) of plasma instabilities $[8-10,14]$. The application of external magnetic fields affects the plasma boundary. The presence of 3D conducting structures may produce further distortion especially during transient phases. Previous studies on JET [11] have shown linear relation between the external $n=1$ magnetic field produced by the EFCCs and the radial displacement of the plasma boundary which predicted significant displacement of the order of $3 \mathrm{~cm} / \mathrm{kA}$ in EFCCs. The studies were performed using the reciprocating edge Langmuir probe measurements and the electron cyclotron emission (ECE) measurements. Studies of 3D distortions have been done on MAST [16], DIII-D [17], NSTX [18] and AUG [19]. They confirmed the existence of 3D distortions due to externally applied fields and nonaxisymmetric plasma response.

The information of the plasma shape 3D deformation is important not only for the correct characterization of the plasma performance and stability analysis, but also to secure safe operations while using EFCC field for different reasons on JET, as the new ITER-like wall is more sensitive to the possible local contact with the plasma than the previous carbon wall. In particular, effect of the $n=2$ perturbation applied for the ELM mitigation on the plasma shape has not been studied before in detail on JET. The recent upgrade of the EFCC power supplies and replacement of one of the coils (which had different specification than three other coils) now allows double the previously applied field. It is worth mentioning that internal modes may also cause a measurable 3D 
distortion of the plasma edge, as the internal displacement due to low-n modes can reach several centimeters [12].

In this work, we use magnetic diagnostics (saddle flux loops measuring radial component of the magnetic field) to obtain information on the radial displacement of the plasma boundary when an external helical magnetic field is applied. A full 3D equilibrium reconstruction using magnetic measurements is currently not available on JET, so a simple method of linear extrapolation of the magnetic data from the sensor location to the plasma edge can be proposed as a first order approximation of the 3D distortion of the plasma edge. Direct measurements of the plasma displacement obtained from the High Resolution Thompson Scattering (HRTS) system at one spatial point are used to calibrate the magnetic measurements at one toroidal position. The experimental scenario and the brief diagnostic description are presented in section 2 . The calibration method and the first results are presented in section 3. Discussion and conclusions are given in section 4.

\section{Experimental scenario and diagnostics}

For the present studies, plasma operations have been used where external helical field with dominant toroidal number $\mathrm{n}=2$ and varying amplitude is applied. The external field is produced by a set of the Error Field Correction coils that are placed in octants 1,3,5,7 outside the first wall. The maximum current in the EFCCs at present is up to $6 \mathrm{kA}$ per turn (multiplied by 16 turns). The layout of the EFCC system is shown in Fig. 1a. Plasma scenario with $\mathrm{I}_{\mathrm{p}}=2 \mathrm{MA}, \mathrm{B}_{0}=2 \mathrm{~T}$ has been chosen for these studies with applied NBI heating with $\mathrm{P}_{\mathrm{NBI}}=8 \mathrm{MW}$. Time traces of several plasma parameters are shown in Fig. 2. The plasma pressure (characterized by the $\beta_{\mathrm{N}}$, Fig. $2 d$ ) is sufficiently low in order not to produce large plasma response that can affect interpretation of the results $[4,11]$. The radial position of the magnetic axis (Fig. 2e) was changed in this pulses to increase the plasma size (not in connection with the application of EFCCs) and has different dynamics with (solid and dashed dotted lines) and without (dashed line) EFCC, which will be discussed later in detail.

The magnetic diagnostic used is an array of flux loops measuring the tangential component of the magnetic field (total magnetic field including plasma contribution and the field from all external sources is measured). The flux loops in octants 1, 3, 5, 7 (14 poloidal position in each octant, see fig. 1b) toroidally coincident with the position of the external coils are complemented by the midplane flux loops (poloidal positions 1,14) in octants 2, 4, 6, 8 for these studies. We estimate plasma boundary displacement from direct magnetic measurements using absolute calibration from the HRTS diagnostic that measures the electron temperature and density at the plasma edge in octant 5 just above the midplane. The line of sight of the HRTS system is coincident with the position of one of the flux loops that is used for calibration (see fig. 1b).

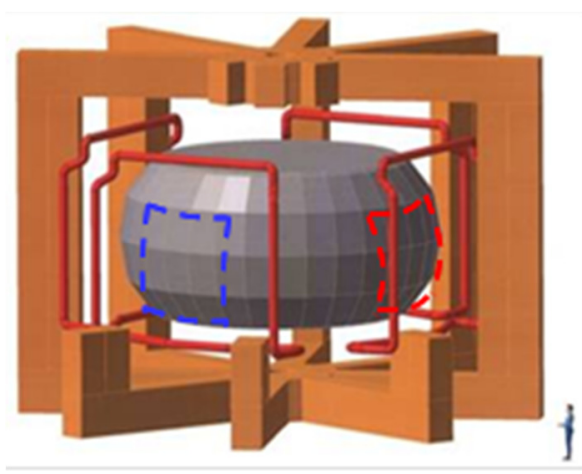

a)

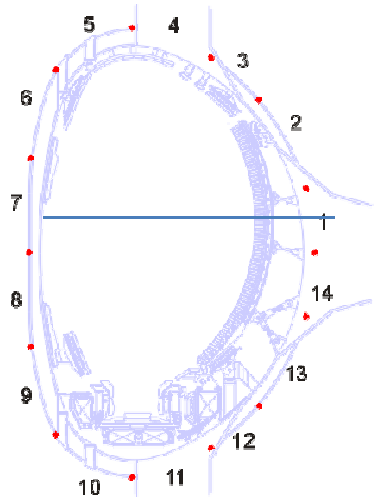

b)

Fig. 1. a) Cartoon illustrating relative position of the external Error Field Correction Coils (solid red) and measuring loops (dashed red and blue, only 2 saddle loops are shown for illustration, combining those above and below the midplane) on JET; b) poloidal cross-section of the JET tokamak with the positions of the saddle loops (marked by dots and numbered), vessel and in-vessel components and the line of sight of the HRTS system (solid line). 

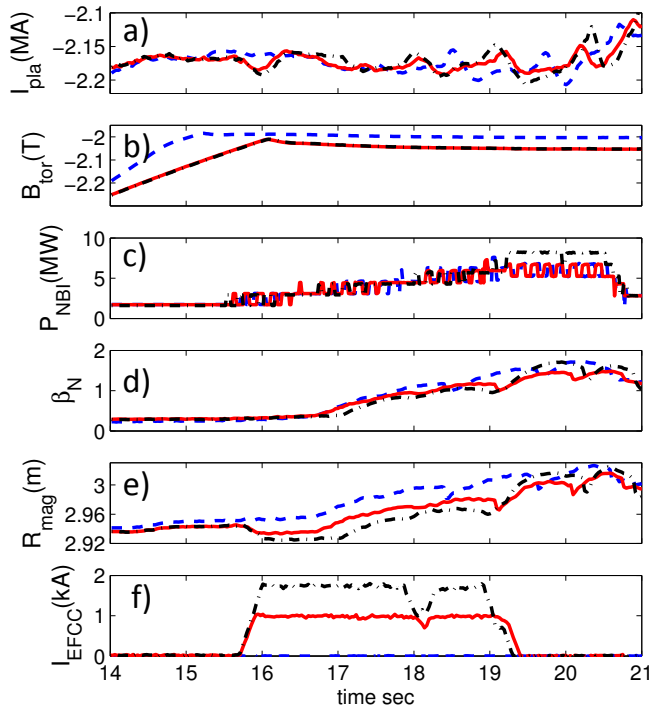

Fig. 2. Time traces of the plasma parameters for the chosen plasma pulses: a) plasma current; b) toroidal magnetic field; c) NBI power; d) normalized beta; e) radial position of the magnetic axis (calculated with EFIT code); f) current in EFCCs. Dashed-pulse 78148, solid-pulse 78149, dashed-dotted-pulse 78151.

The direct measurements of the plasma boundary displacement are obtained using HRTS diagnostic by following the time evolution of the edge density profile as shown on fig. 3 for the pulse \#78151 with applied $\mathrm{I}_{\mathrm{EFCC}}=1.75 \mathrm{kA}$. The procedure to determine the pedestal position takes into consideration the HRTS instrument function as described in [13].
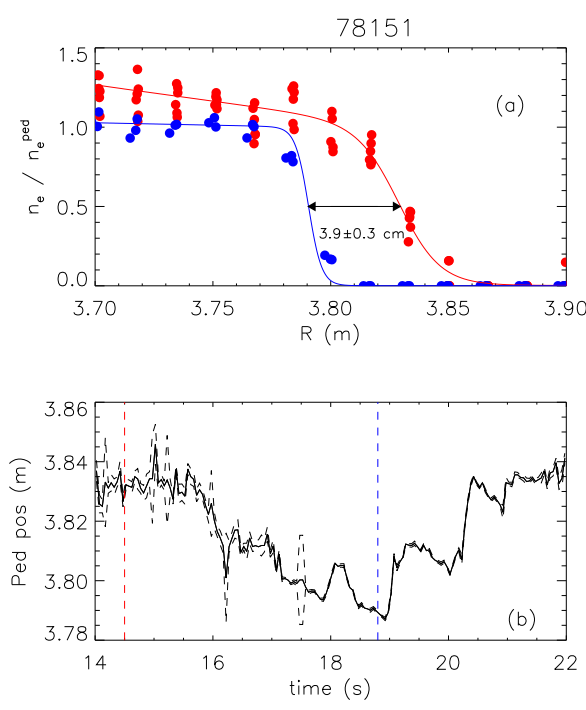

Fig. 3. Direct measurements of the plasma boundary displacement using HRTS diagnostic: a) edge electron density profiles $t=14.2 \mathrm{sec}$ (red) and $18.7 \mathrm{sec}$ (blue); b) time evolution of the pedestal position measured approximately at the middle of pedestal (marked by the horizontal arrows in frame (a)). The dashed lines in Fig. $3 b$ correspond to the time points shown in frame (a).

The time evolution of the EFCC current, plasma boundary displacement measured by the HRTS (scaled to have zero displacement before application of the EFCC produced magnetic field) and the radial magnetic flux measured by the coincident flux loop for the shots with different values of 
the EFCC current are shown in Fig. 4. The effect of the field produced by EFCC coils on the plasma boundary is clearly seen in the kinetic measurements, Fig. 4b (comparison of displacement for the cases with and without external field applied). It is also seen that apart from EFCC effect there is an offset effect from other sources. The dominant contribution to the offset is due to the plasma shape control system (preprogrammed outward shift is applied for the times $t>17.0 \mathrm{sec}$ ) and its effect is not of direct interest for these studies (as it has $n=0$ structure), although reaction of the JET control system on the applied EFCC field is important for operations. The offset will be further removed from kinetic and magnetic measurements assuming the same shape control scheme for these shots and subtracting the signal corresponding to the case $\mathrm{I}_{\mathrm{EFCC}}=0$ (dashed line on Fig. 4) from other signals. Some rapid change of the EFCC current signal is seen around $t=18 \mathrm{sec}$. This change is attributed to the sudden loss of control of the EFCC power supply and is not important for the present studies, although it clearly results in a change in the edge displacement.
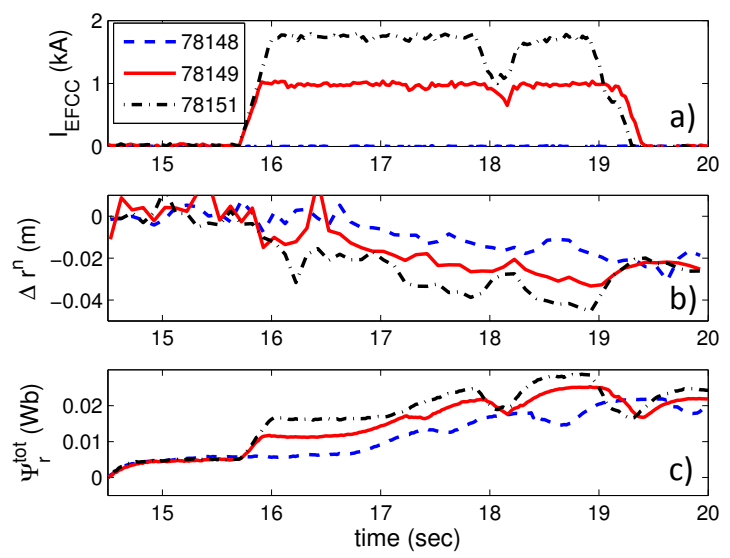

Fig. 4. Experimental signals used for the studies: a) EFCC current (dashed- $I_{E F C C}=0$, solid$I_{E F C C}=1 \mathrm{kA}$, dashed-dotted $\left.-I_{E F C C}=1.75 \mathrm{kA}\right) ; b$ ) plasma boundary displacement obtained from the edge density measured by HRTS; $c$ ) radial magnetic field measured by the flux loop coincident with the HRTS position.

\section{Calibration method and first results}

In order to use magnetic measurements for the plasma boundary displacement studies, a calibration should be performed finding the transformation rule between the measured magnetic field and the displacement value. As the majority of the saddle loops (excluding those in the divertor region) are within a few centimeters from the edge of the plasma, a linear extrapolation has been used in these studies, which may not be perfect. The work is on-going to study possible limitations of this approximation, both in axisymmetric and 3D cases. Qualitative comparison of the kinetic and magnetic signals is performed first after subtraction of the $n=0$ part. This comparison is done in order to estimate the efficiency of the performed subtraction as in principle such offset may vary from pulse to pulse. However, the fact that both the magnetic and kinetic data goes down to the zero-EFCC level after the end of the EFCC pulse (Fig. $4 \mathrm{t}=19.2 \mathrm{sec}$ ) makes this way of the offset subtraction reasonable (see more in the Discussion section). The result of the comparison is shown in Fig. 5. The effect of the helical external magnetic field on the plasma boundary is more evident after the offset subtraction (Fig. 5b compared to Fig. 4b). It is seen that the general shape of the kinetic and magnetic measurements follows well the shape of the EFCC current validating subtraction of $n=0$ part. The correlation between the kinetic and magnetic data is seen even after the end of the EFCC pulse ( $t>$ $19.2 \mathrm{sec}$ ), when displacement is caused by other reasons, not discussed in this paper. However, it is clear that what may be seen as a noise on the signals actually represents the real edge displacement, and of a measurable value, as kinetic and magnetic measurements are completely independent.

The quantitative part of the calibration consists of finding the relation between the kinetic and magnetic measurements for different values of the EFCC current (pulses with $\mathrm{I}_{\mathrm{EFCC}} \leq 3 \mathrm{kA}$ are used, with the plasma boundary displacement detected by the HRTS system $\Delta \mathrm{r}_{\text {kin }} \leq 5 \mathrm{~cm}$ ). The results are shown in Fig. 6a. The relation is taken for the flat-top phase of the EFCC current and the 
error bars shown on the figure represent deviation of the measurements (kinetic or magnetic) from the mean value. It is seen that the experimentally obtained relation (stars) is well approximated by the linear fit (dashed line). As a final step the comparison of the plasma boundary displacement evolution obtained by the HRTS and that reconstructed using magnetic measurements (using the results of the linear fit) is done. It is shown in Fig. 6b. It is seen that good agreement is obtained between measured and reconstructed displacement evolution. Although such relation in principle may vary for different locations, we use it as a first approximation, and it is planned to check the calibration at different locations by using other diagnostics.

The spatial dependence of the radial plasma boundary displacement can now be studied applying the results of the calibration to the set of the magnetic sensors distributed in the toroidal and poloidal directions. In Fig. 7 the spatial dependence of the radial displacement on the poloidal and toroidal angles is shown for the cases of two different EFCC current values. The $n=2$ displacement component is seen in the toroidal direction (as expected). Dominant $\mathrm{m}=1$ component is observed in the poloidal direction, well correlated with the poloidal spectrum of EFCCs (not shown). It should be noted that the plasma response to the external field was assumed to be low in these experiments. It is seen also that the displacement is not symmetric, being larger at the outboard side (poloidal angle values around $\theta=0$ ). The dependence on the EFCC current is also seen (radial displacement is larger for the larger EFCC current value), however the discussed above non-linearity is present and should be taken into account in future more detailed studies which should include more diagnostics spread both in the toroidal and poloidal directions (e.g. CXRS, reciprocating probe, ECE, SXR etc.)

The dependence on the poloidal and toroidal angles can be generalized using magnetic measurements obtained by the array of magnetic sensors described in section 2 (i.e. $4 \times 14$ flux loops). The 3D map of the radial displacement of the plasma boundary for the case of $\mathrm{I}_{\mathrm{EFCC}}=1.75 \mathrm{kA}$ is shown in Fig. 8. Interpolation of the data in-between measurement points is performed for poloidal and toroidal angles. The 'offset' is removed from the measurements to have zero value just before EFCC is applied. Dominant $\mathrm{n}=2$ (along toroidal angle) and $\mathrm{m}=1$ (along poloidal angle) components of the displacement are again clearly observed. The maximum absolute value of the plasma displacement is around $3.0 \mathrm{~cm}$ (for $1.75 \mathrm{kA}$ EFCC current) that is in agreement with the previously obtained results [11], assuming n-dependence. Such a map may now be used by operators to predict possible plasmawall contact when EFCCs are applied. In the future, this data can be complimented by the RFA measurements [4], which may be used to predict the plasma response amplification as well as the "vacuum" field displacement effect and so show possible limitations of the use of EFCCs at higher heating powers.
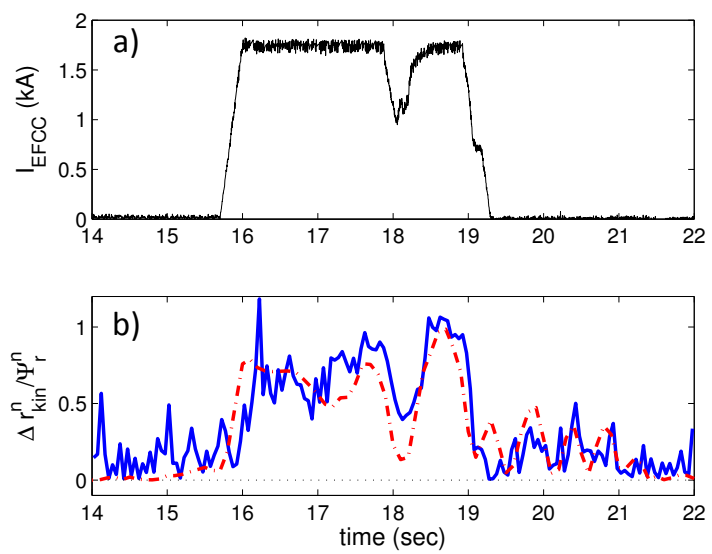

Fig.5. Comparison of the normalized displacement of the plasma boundary measured by the kinetic and magnetic diagnostics. Comparison is shown for one value of the EFCC current $\left(I_{E F C C}=1.75 \mathrm{kA}\right)$. a) EFCC current; b) normalized displacement measured by the kinetic (solid) and magnetic (dasheddotted) diagnostics. 

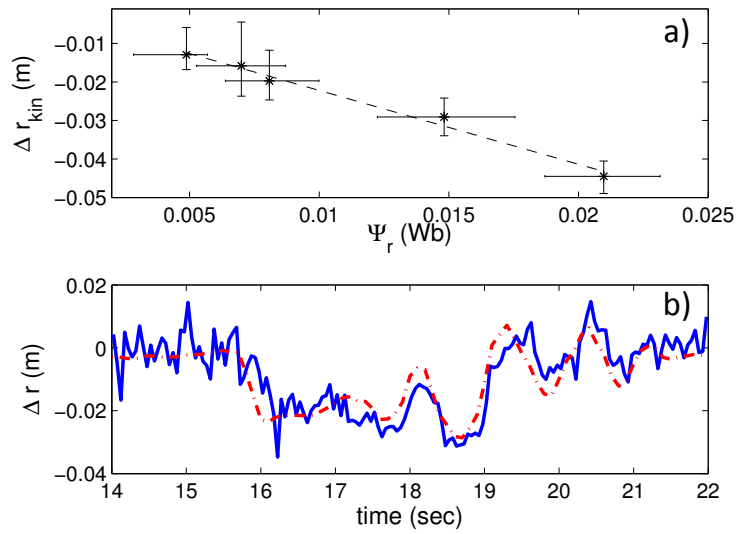

Fig. 6. Results of the calibration of the magnetic measurements using the (absolute) kinetic measurements. a) dependence of the plasma boundary displacement obtained from the kinetic measurement on that obtained from magnetic measurements (stars) and linear fit (dashed line); $b$ ) reconstruction of the plasma boundary displacement obtained from the kinetic measurements for $I_{E F C C}=1.75 \mathrm{kA}$ (solid) using magnetic measurements and the coefficients of the linear fit (dasheddotted).
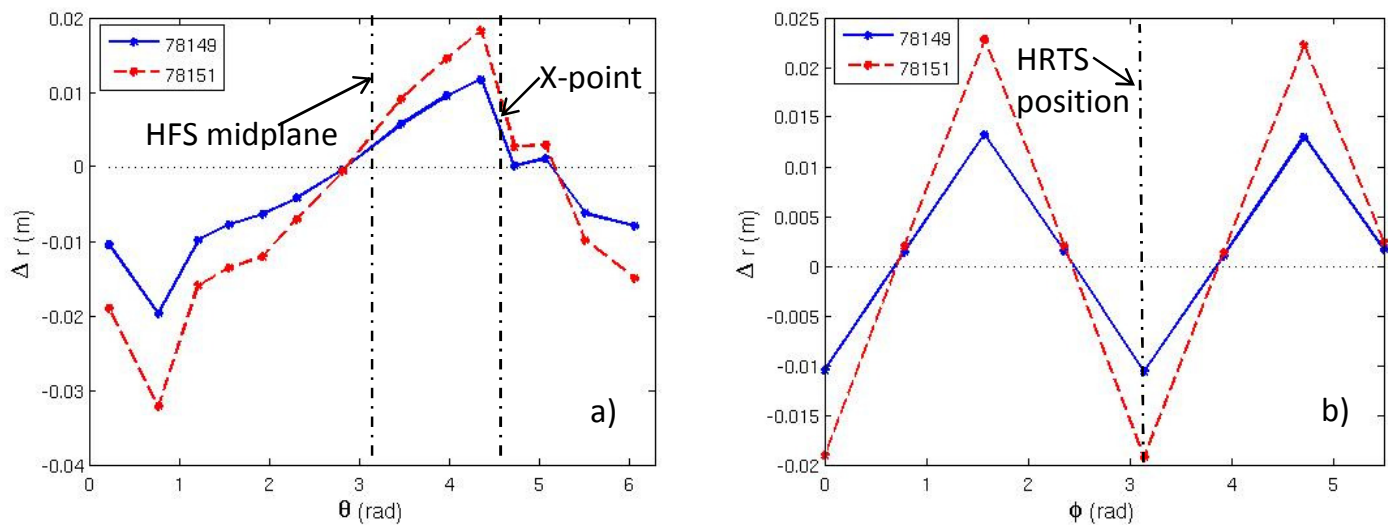

Fig. 7. Spatial dependence of the plasma boundary displacement for different values of EFCC current calculated for one time point $(t=16.6 \mathrm{sec})$ using flux loop array and linear model for conversion of the magnetic signal to the displacement: a) poloidal dependence calculated for octant $5(\phi \approx \pi)$, poloidal angle $\theta=0$ coresponds to the low field side (LFS) midplane, poloidal angles of the high field side

(HFS) midplane $(\theta \approx \pi)$ and $X$-point $(\theta \approx 3 \pi / 2)$ are marked by the dashed-dotted lines; $b)$ toroidal dependence calculated for the one polodal angle (poloidal angle of the first sensor in poloidal direction), toroidal position of HRTS system is marked by dashed-dotted line. Solid $-I_{E F C C}=1 \mathrm{kA}$, dashed $-I_{E F C C}=1.75 \mathrm{kA}$. Geometrical centers of the sensor coils (in poloidal and totoidal directions) are marked by the stars. In the case b) stars correspond also to the toroidal angles of the octant's positions ( $\phi=0$ corresponds to octant 1 and last point corresponds to octant 8). 


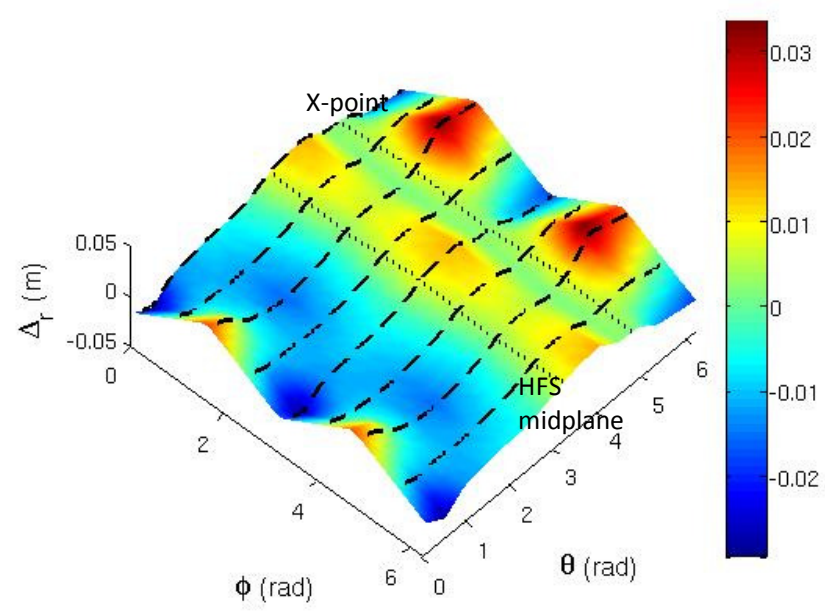

Fig. 8. The dependence of the plasma boundary displacement $\Delta_{r}$ on the toroidal $(\phi)$ and poloidal $(\theta)$ angles calculated for one time point $(t=16.5 \mathrm{sec})$ for the case $I_{E F C C}=1.75 \mathrm{kA}$. Dashed lines mark toroidal angles of octant's positions, dotted lines - poloidal angles of the HFS midplane and X-point, $\theta=0$ corresponds to the LFS midplane, $\phi=0$ corresponds to the geometrical center of octant 1 .

\section{Discussion and Summary}

A simplified method to study radial plasma boundary displacement caused by the external magnetic perturbations using the magnetic measurements was proposed in this paper. A recent upgrade of the JET magnetic diagnostics to allow the full coverage of the torus makes this method particularly useful, since the plasma boundary shape both poloidally and toroidally can be studied.

As discussed above, the note on the possible plasma response effect on the obtained results should be made. It was mentioned in the previous studies, that the plasma response behavior becomes non-monotonic either near the stability boundaries of the MHD modes [4] or near the modelocking threshold when increasing the value of the externally applied field [11]. Non-monotonic plasma response therefore will change the relation between the applied and measured magnetic field in a complex and non-linear way as the spatial distribution of the plasma response will depend on the stability of particular modes. On the other hand it is not clear whether the relation between measured magnetic field and directly measured radial plasma boundary displacement (that is used here) will change significantly as the flux structure outside the plasma is affected by other than the plasma influence, i.e. by the metal structures and vacuum fields. This question remains the subject of further studies.

Several assumptions and simplifications were made in the present work that could affect accuracy of the results. The first assumption is that the effect of the plasma shape control system is the same for all shots studied. As discussed, such assumption allowed simple subtraction of the 'reference' signal to remove the unwanted control system contribution. In order to evaluate the applicability of such method Fourier transform in toroidal direction is performed for the shots discussed above (same shots as on Fig. 4) using raw magnetic measurements i.e. without subtraction the flux from the $\mathrm{I}_{\mathrm{EFCC}}=0$ case. The amplitudes of the obtained Fourier components are shown on Fig. 9. 

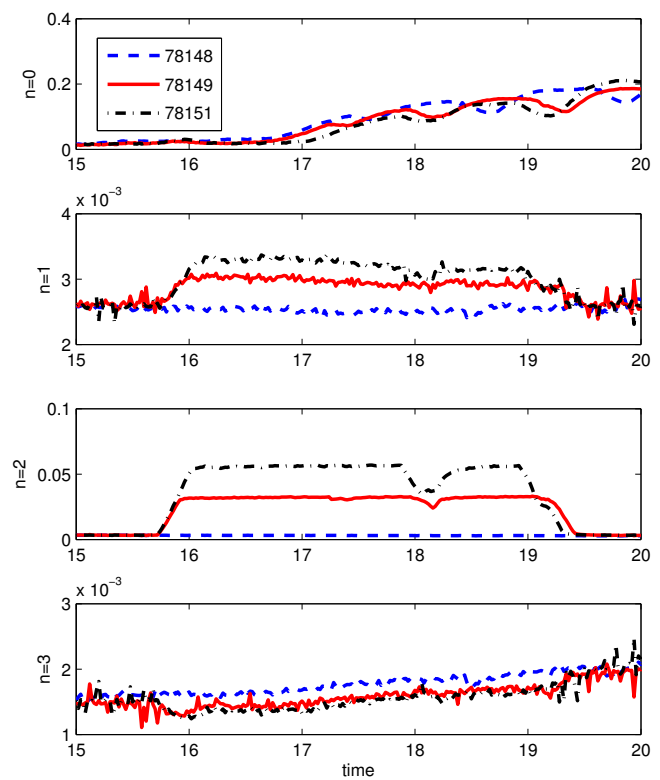

Fig. 9. Toroidal Fourier components of the raw magnetic measurements. Dashed$I_{E F C C}=0 \mathrm{kA}$, solid $I_{E F C C}=1.0 \mathrm{kA}$, dashed-dotted $I_{E F C C}=1.75 \mathrm{kA}$.

It is seen that the dominant Fourier components are $n=0$ and $n=2$. While $n=2$ component correspond to the EFCC current, $\mathrm{n}=0$ component has different behavior that is believed to be caused by the shape controller. It is seen that for the times $t \leq 17 \mathrm{sec}$ the effect from the shape controller is the same for all cases. For the times $t>17 \mathrm{sec}$ the effect is slightly different for each case. The conclusion that can be drown is that for the times later than $17 \mathrm{sec}$ uncertainties in the displacement calculations are expected if using simple subtraction method (the spatial distribution presented on Figs. 7,8 are calculated for $\mathrm{t}=16.5 \mathrm{sec}$ ). In reality the shape control system effect could be different for different shots (as the plasma parameters are not absolutely the same in each pulse) and therefore the effect of the shape control system on the results should also be studied. A number of specific experiments have been performed on JET during first operations with the full-metal wall to find empirically the best way of controlling the plasma shape and position in the presence of the non-axisymmetric magnetic fields and this work will be continued for different scenarios.

Another assumption made is that the linear coefficient obtained during the calibration process is applicable for all magnetic sensors. As the calibration was done using the measurements at the plasma edge (HRTS) and measurements taken at some distance from the plasma (magnetic sensors) the distance between the plasma and the saddle coils is indirectly present in the coefficient calculations. As the calibration was done just for one sensor and the distance between sensors and the plasma boundary is different along the poloidal direction the calibration coefficient value should be refined for the other sensors. As mentioned above, this can be done either by using direct measurements of the radial plasma displacement taken at different spatial locations or by using full 3D magnetic reconstruction [15]. The presented results are more reliable near the midplane, where the HRTS measurements have been done.

As a last note, the presented results are obtained for the plasma operations with the carbon wall. First kinetic measurements of the plasma boundary distortion for the ITER-like (metal) wall (not shown here) give $\Delta \mathrm{r}_{\mathrm{kin}} \approx 6 \mathrm{~cm}$ for $\mathrm{I}_{\mathrm{EFCC}}=3 \mathrm{kA}$ that is similar to the results shown here (see Fig $6 a)$.

In conclusion, studies of the radial plasma boundary displacement caused by the external helical magnetic field have been performed. The magnetic measurements calibrated by the direct kinetic measurements from HRTS diagnostics were used. Linear dependence of the radial plasma displacement and the radial magnetic field is found that is in agreement with the previous studies. The value of the plasma displacement is around $3 \mathrm{~cm}$ for $\mathrm{I}_{\mathrm{EFCC}}=1.75 \mathrm{kA}$ and is not symmetric around the poloidal direction, being larger at the outboard side. Such displacement caused by the $n=2$ 
field is in a reasonable qualitative agreement with that measured when $n=1$ field was applied, taking into account the toroidal number effect. Further studies are required in order to clarify the effect of the plasma response and also to improve the accuracy of the used method.

\section{Acknowledgments.}

Authors would like to acknowledge JET session leaders who performed experiments,

Diogo Alves, who created the EFCC controller and provided settings of EFCC currents, and the contribution of Joanne Flanagan and Mark Kampenaars to the discussions related to the HRTS diagnostics and Peter Lomas for pointing out on the deliberate plasma shift in the analysed pulses. This work was supported by EURATOM and carried out within the framework of the European Fusion Development Agreement. The views and opinions expressed herein do not necessarily reflect those of the European Commission.

\section{References}

1. Garofalo A. M. et al, 2002 Phys. Plasmas 91997

2. Gryaznevich M. P. et al, 2003 Bull. Am. Phys. Soc 48307

3. Liu Y. et al, 2009 Plasma Phys. Control. Fusion 51115005

4. Gryaznevich M. et al, 2012 Nucl. Fusion 52083018

5. Gregoratto D. et al, 2005 Phys. Plasmas 12092510

6. Olofsson K. E. J., Brunsell P. R. and Drake J.R., 2012 Fus. Eng. Design 851926

7. Strait E. J. et al, 2003 Nucl. Fusion 43430

8. Garofalo A.M. et al, 2007 Nucl. Fusion 47 1121-1130

9. Brunsell P. R. et al, 2004 Phys. Rev. Lett. 93225001

10. Suttrop W. et al, 2011 Plasma Phys. Control. Fusion 53124014

11. Chapman I.T. et al, 2007 Nucl. Fusion 47 L36

12. Buratti P. et al, in Fusion Energy 2010 (Proc. 23rd Int. Conf. Daejeon, 2010) (Vienna: IAEA) CD-ROM file (EXS/P5-02) http://wwwnaweb.iaea.org/napc/physics/FEC/FEC2010/html/index.htm

13. Frassinetti L. et al, 2012 Rev. Sci. Instrum. 83013506

14. Liang Y. et al, 2010 Nucl. Fusion $\mathbf{5 0} 025013$

15. Hanson J. et al, 2009 Nucl. Fusion 49075031.

16. Chapman I.T. et al, 2012 Plasma Phys. Control. Fusion 54105013

17. Lanctot M. J. et al 2011 Phys. Plasmas 18056121

18. Canik J. et al, 2012 Nucl. Fusion 52054004

19. Fischer R et al, 2011 Effect of non-axisymmetric magnetic perturbations on profiles at ASDEX Upgrade. 38th EPS Conf. on Plasma Physics (Strasbourg, France) P1.072(http://ocs.ciemat.es/EPS2011PAP/pdf/P1.072.pdf) 